Pegalajar Palomino, M.C. (2021). Implicaciones de la gamificación en Educación Superior: una revisión sistemáticá sobre la percepción del estudiante. Revista de Investigación Educativa, 39(1), 169-188.

DOI: http://dx.doi.org/10.6018/rie.419481

\title{
Implicaciones de la gamificación en Educación Superior: una revisión sistemática sobre la percepción del estudiante
}

\section{Implications of gamification in Higher Education: a systematic review of student perception}

\author{
$M^{a}$ del Carmen Pegalajar Palomino \\ Universidad de Jaén
}

\begin{abstract}
Resumen
La gamificación se entiende como una estrategia metodológica innovadora que incorpora las estrategias, dinámicas, mecánicas y elementos propios del juego al proceso de enseñanzaaprendizaje. Este trabajo realiza una revisión sistemática para identificar los principales hallazgos reportados en la literatura científica sobre la percepción del estudiante universitario hacia la práctica de estrategias de gamificación en su proceso de aprendizaje. La búsqueda documental se ha llevado a cabo a partir de la triangulación de las publicaciones incluidas en las bases de datos "Web of Science" y "Scopus" durante el período 2010-2019. Tras aplicar los criterios de selección y calidad, se han identificado un total de 20 estudios como muestra final. Los resultados demuestran el interés creciente de la comunidad científica por plantear trabajos sobre gamificación en Educación Superior. Además, se corrobora una predisposición favorable en el alumnado hacia el desarrollo de experiencias didácticas innovadoras basadas en la gamificación. Entre sus potencialidades, destaca el aumento de la motivación, el interés y la participación del alumnado en el proceso de enseñanza-aprendizaje, así como la mejora del rendimiento académico y el desarrollo de las habilidades y competencias necesarias para su desarrollo profesional.

Palabras clave: gamificación; Educación Superior; rol del estudiante; aprendizaje.
\end{abstract}

\footnotetext{
Correspondencia: $\mathrm{M}^{\mathrm{a}}$ del Carmen Pegalajar Palomino, mcpegala@ujaen.es Universidad de Jaén. Campus "Las Lagunillas", s/n. Facultad de Humanidades y Ciencias de la Educación. Departamento de Pedagogía. C.P. 23071. Jaén (España).
} 


\begin{abstract}
Gamification is understood as an innovative methodological strategy that incorporates the strategies, dynamics, mechanics and elements of the game into the teaching-learning process. This work provides a systematic review which identifies the main findings reported in the scientific literature on the perception of the university student towards the practice of gamification strategies in their learning process. The documentary search carried out was based on the triangulation of the publications included in the "Web of Science" and "Scopus" databases during the period 2010-2019. After applying the selection and quality criteria, a total of 20 studies have been identified as the final sample. The results demonstrate the growing interest of the scientific community to propose works on gamification in Higher Education. Furthermore, a favorable predisposition of the students towards the development of innovative didactic experiences based on gamification is corroborated. Among its potential, the increase in motivation, interest and participation of students in the teaching-learning process stands out, along with the improvement of their academic performance and the development of the skills and competences necessary for their professional development.
\end{abstract}

Keywords: Gamification; Higher Education; student role; learning.

\title{
Introducción
}

El proceso europeo instituido en la Educación Superior ha supuesto una preocupación por la búsqueda y el mantenimiento de la calidad educativa, con la intención de garantizar unos mejores resultados de aprendizaje en el estudiante (Herrera, Souza \& Soares, 2018). Ante este proceso de cambio, surge la gamificación entendida como una estrategia didáctica innovadora que implica la incorporación de dinámicas o mecanismos de juego en entornos o procesos que no constituyen un juego en sí mismos (Alsawaier, 2018). Por su parte, la estrategia centrada en el Aprendizaje Basado en Juegos (Game-Based Learning, GBL) trata de integrar el juego para producir determinados comportamientos según los objetivos educativos (Pivec \& Dziabenko, 2004). A diferencia de la gamificación, se crea el contenido a enseñar como elemento transversal de la inmersión (Torres, Ramírez \& Romero, 2018).

Distintas investigaciones (Borrego, Fernández, Blanes \& Robles, 2017; Ke, 2014; Pérez \& Almela, 2018) destacan la eficacia de trasladar al contexto educativo nuevas metodologías de aprendizaje, ofreciendo posibilidades de feedback inmediato, informes de progreso en el estudiante y recompensas que motivan dicho avance (Bodnar, Anastasio, Enszer \& Burkey, 2016).

Hanus y Fox (2015) exponen cómo la gamificación establece un vínculo entre el estudiante y el contenido desde una perspectiva diferente, con la intención de comprender mejor el conocimiento o perfeccionar determinadas habilidades que favorezcan su empleabilidad (Dascalu, Tesila \& Nedelcu, 2016) como puedan ser: creatividad, resolución de problemas, comunicación y colaboración, toma de decisiones, alfabetización, etc. (Moffat, Farrell, Gardiner, McCulloch \& Fairlie, 2015).

La gamificación permite que el alumnado participe en la creación de una comunidad de aprendizaje, pudiendo experimentar con libertad y aprender de los errores en un entorno agradable (Brull \& Finlayson, 2016). Además, y según Ardila (2019) favorece 
que las actividades evaluativas pierdan su carácter punitivo, estableciéndose un proceso didáctico basado en la competitividad y cooperación mediante un aprendizaje basado en problemas y por descubrimiento. De este modo, el estudiante se enfrenta a la tarea no como un trabajo, sino como una actividad lúdica que conecta con su centro de interés (Sánchez, Cañada \& Dávila, 2017), lo que favorece su compromiso hacia el estudio (Abdul \& Felicia, 2015; Huang, Hew \& Lo, 2019) y el desarrollo de un aprendizaje más significativo (Prieto, 2018).

Además, uno de los mayores atractivos del aprendizaje gamificado es el uso de las Tecnologías de la Información y la Comunicación (en adelante, TIC) (Gay \& Burbridge, 2016; Howel, Tseng \& Colorado, 2017; Medvedovska, Skarlupina \& Turchyna, 2016), las cuales permiten en el estudiante una mayor toma de conciencia sobre la regulación de su experiencia de aprendizaje (Chaiyo \& Nokham, 2017).

No obstante, Rodríguez-Hoyos y Joao (2013) subrayan cómo los juegos y videojuegos no suponen "per se" un recurso potenciador del conocimiento en el estudiante, siendo lo más importante la metodología empleada para el uso del juego en el proceso didáctico. El profesorado debe prestar especial atención en el diseño, planteamiento y la puesta en práctica de esta estrategia metodológica, pues condiciona los resultados esperados en el estudiante (Contreras \& Eguia, 2016; Oliva, 2017). En este sentido, el estudio de Aldemir, Celik y Kaplan (2018) revela el alto grado de aceptación de la gamificación por parte del estudiante si el juego es diseñado de manera adecuada. Por tanto, no se debe confundir la gamificación con la simple adaptación de un juego al contenido docente, siendo necesario el desarrollo de incentivos intrínsecos en el estudiante (Kokkalia, Drigas, Roussos \& Economou, 2017).

La investigación sobre la gamificación es una tendencia relativamente reciente, habiéndose publicado en los últimos años distintos trabajos de revisión (Dicheva, Dichev, Agre \& Angelova, 2015; Lozada \& Betancur, 2017; Pascuas, Vargas \& Muñoz, 2017) que exploran la producción bibliográfica de esta área considerada de interés, así como su impacto en el contexto educativo. Sin embargo, y dada la importancia de examinar los elementos que favorecen o dificultan la implantación de esta estrategia metodológica en el aula universitaria, este trabajo pretende dar respuesta al siguiente interrogante: ¿Qué hallazgos reporta la literatura científica sobre la percepción del estudiante universitario hacia los procesos de aprendizaje basados en la gamificación?

\section{Método}

Esta revisión sistemática se ha desarrollado atendiendo a los principios de la declaración PRISMA (Urrútia \& Bonfill, 2010) y las pautas metodológicas estandarizadas propuestas para la elaboración de revisiones sistemáticas de calidad (Alexander, 2020).

\section{Objetivos}

Este trabajo pretende desarrollar una revisión sistemática de las investigaciones empíricas publicadas en las bases de datos "Web of Science" (WoS) y "Scopus" durante el período 2010-2019. Además, y de modo más concreto, se plantean los siguientes objetivos específicos: 
- 1: Describir la producción científica publicada sobre esta temática en las bases de datos seleccionadas durante el período 2010-2019.

- 2: Sintetizar la evidencia reportada en la literatura científica sobre esta temática objeto de estudio.

\section{Búsqueda en base de datos}

Para este trabajo se ha seleccionado como principal motor de búsqueda la base de datos "Web of Science" (WoS). . Además, se ha utilizado la base de datos "Scopus" para contrastar y complementar la información obtenida en la base de datos principal.

Para la búsqueda de documentos, se han utilizado como palabras clave los siguientes términos, tanto en español como en inglés: “Gamificación"), "Aprendizaje basado en el juego", "Educación Superior", "Universidad”, "Aprendizaje centrado en el estudiante” y como operadores boleanos "AND" y "OR". De este modo, la ecuación definitiva de búsqueda en español ha sido: ("Gamificación" OR "Aprendizaje basado en juego") AND ("Aprendizaje centrado en el estudiante") AND ("Educación Superior" OR "Universidad"). Por su parte, los términos de búsqueda en inglés se corresponden con: ("Gamification" OR "Gamed Based Learning") AND ("Student Centered Learning") AND ("Higher education" OR “University).

No obstante, y con la intención de acotar el número de publicaciones inicialmente encontradas en ambos repositorios, se han delimitado una serie de criterios de inclusión y exclusión para su selección, siendo éstos (Tabla 1):

Tabla 1

Criterios de inclusión y exclusión

\section{Criterios de inclusión Criterios de exclusión}

Investigaciones descriptivas hacia experiencias de Actas de congresos, libros, capítulos de aprendizaje basadas en la gamificación en Educación libros

Superior

Período: 2010-2019

Todos los contextos geográficos

Idioma: español o inglés

Áreas de conocimiento: Ciencias Sociales e Investig-

ación educativa

Revistas con indicios de calidad y revisión por pares

Documentos en abierto

\section{Estudios teóricos o de revisión}

Estudios descriptivos sobre experiencias de gamificación para otras etapas educativas distintas a la Educación Superior
Publicaciones duplicadas

\section{Categorización de las publicaciones}

La puesta en marcha del protocolo de búsqueda en las bases de datos ha identificado un total de 1245 publicaciones como documentos potenciales. El elevado número de 
trabajos hace necesario refinar esta primera muestra aplicando los criterios de selección anteriormente descritos. Las principales razones para excluir las investigaciones inicialmente identificadas han sido: estudios que desarrollan experiencias de gamificación para estudiantes de educación básica (principalmente, educación secundaria) o publicaciones de tipo teórico.

Esta tarea ha sido desarrollada por tres expertos independientes, quienes han revisado el título y resumen de cada uno de los estudios, dando lugar a la reducción de la muestra a 132 publicaciones Las codificaciones realizadas por los investigadores para cada uno de los estudios han revelado un alto grado de acuerdo (91\% de las publicaciones revisadas). Tan sólo se produjeron discrepancias en las codificaciones de 12 publicaciones, por lo que los investigadores han revisado de nuevo la documentación para llegar a un acuerdo consensuado.

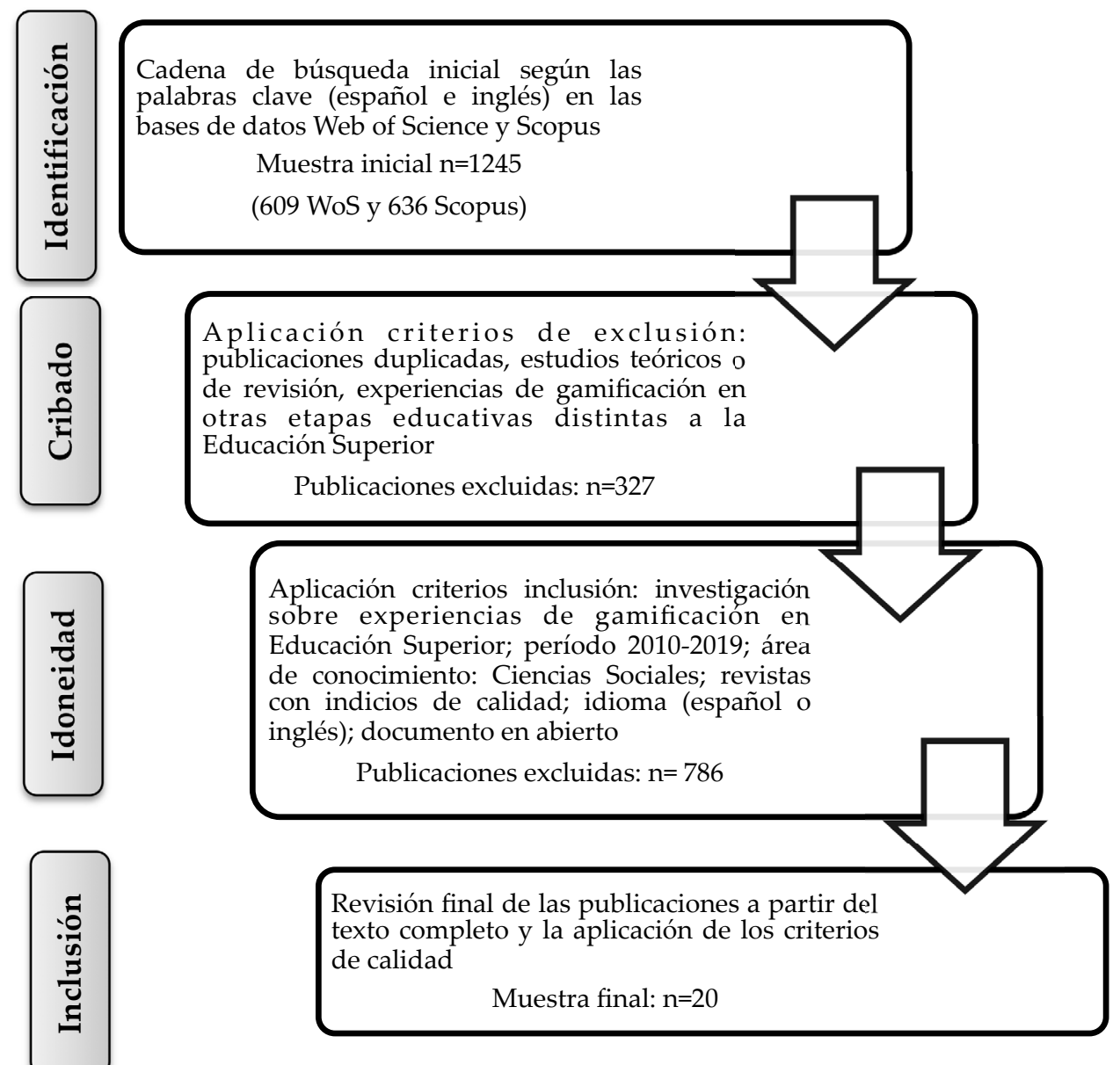

Figura 1. Diagrama de flujo para el proceso de selección de la muestra 
Por último, se ha llevado a cabo una revisión final de cada publicación a partir de la lectura detallada del texto completo para comprobar criterios de calidad. Así pues, la muestra final de este estudio está compuesta por 20 publicaciones. La Figura 1 muestra el diagrama de flujo que describe el proceso seguido para la búsqueda de las publicaciones y su refinamiento hasta llegar a la concreción de la muestra definitiva.

\section{Análisis global de los resultados}

Para la organización de las publicaciones obtenidas en cada base de datos se ha utilizado el software de gestión de publicaciones "Mendeley". El análisis de la muestra definitiva se ha realizado mediante una plantilla de datos elaborada con el software Microsoft Excel.

\section{Resultados}

\section{Análisis de la producción científica}

La evolución de la producción científica inicialmente filtrada en las bases de datos consultadas $(n=1245)$ refleja el crecimiento ascendente de publicaciones sobre gamificación en Educación Superior a partir de 2011, siendo el período comprendido entre 2017-2019 dónde más trabajos sobre esta temática se han publicado, con un $67.61 \%$ de la producción total, tal y como se muestra en la Figura 2.

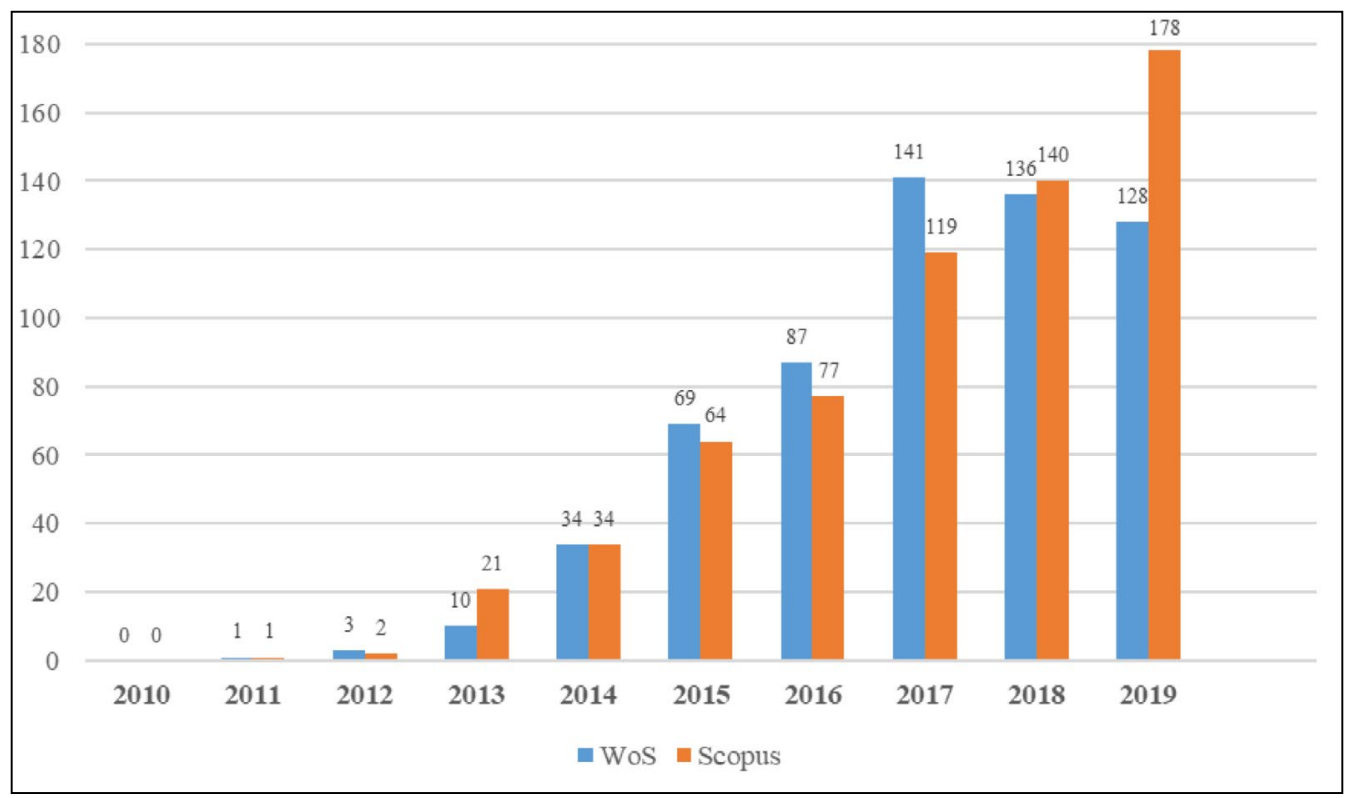

Figura 2. Evolución de las publicaciones de 2010 a 2019 (Fuente: WoS y Scopus). 
Tal y como se expone en la Tabla 2, la mayor parte de los trabajos han sido divulgados en revistas científicas en el año 2019 (n=15), tratándose de publicaciones compartidas por varios autores. Los estudios seleccionados provienen de diferentes países, reflejando la relevancia e importancia de la temática a nivel mundial. Dichos estudios incluyen el término "gamificación" como palabra clave para su identificación, aunque también añaden otros términos que demuestran su relación con el proceso de enseñanza-aprendizaje, como pueda ser: "motivación", "enseñanza y aprendizaje”, "pedagogía”, "estudiantes”, "aprendizaje cooperativo”, "metodologías activas”, "innovación educativa", "diseño de juegos", etc.

Las lenguas inglesa y española son las utilizadas en las publicaciones, incorporadas en revistas científicas que siguen un riguroso proceso de publicación a partir de la revisión por pares. No obstante, España es el país que mayor número de estudios científicos ha publicado sobre esta temática objeto de estudio $(n=6)$, seguido de Malasia $(\mathrm{n}=3)$, Reino Unido y Ucrania $(\mathrm{n}=2)$, respectivamente. En cuanto al número de citas de las publicaciones, cabe destacar el trabajo desarrollado por Pettit, McCoy, Kinney y Schwart (2015) publicado en la revista "BMC Medical Education" con un total de 19 citas.

Las estrategias de gamificación se han desarrollado en titulaciones adscritas a varias áreas de conocimiento, fundamentalmente las de Ciencias Sociales y Jurídicas (en las titulaciones de Ciencias de la Educación, Ciencias Económicas y Empresariales), Arte y Humanidades (Filología), Ingeniería y Arquitectura (Ingeniería Informática) y Ciencias de la Salud (Medicina). Tras el análisis detallado de frecuencias por áreas, la que mayor frecuencia registra es el área de Ingeniería Informática $(n=6)$, seguida de Medicina y Filología Inglesa $(n=3)$ y Ciencias de la Actividad Física y del Deporte, Estadística, Relaciones Laborales, Turismo, Educación Primaria $(n=2)$ y, finalmente, Psicología y Humanidades $(n=1)$ respectivamente.

En cuanto a las herramientas de gamificación propuestas en las distintas investigaciones, muchas de las publicaciones revisadas incluyen como sistemas de gamificación aplicaciones TIC basadas en la realización de cuestionarios de elección múltiple, como puedan ser: Kahoot!, Socrative, Quizziz, CelebritiEdu, TurningPoint, etc.

En otros tipos de trabajos se apuesta por el desarrollo de juegos de simulación, narrativa y realidad virtual que permitan ubicar al estudiante en situaciones reales a las que podrán encontrar en su futura práctica profesional. Incluso, y centrados en el desarrollo profesional del estudiante, este trabajo incluye una investigación que apuesta por el desarrollo de experiencias emprendedoras, ofreciéndole la posibilidad ejercer las funciones como directivo comercial y desarrollar una idea de negocio. Finalmente, otras experiencias plantean como estrategia de aprendizaje la realización de juegos de roles con los que facilitar la adquisición de competencias interpersonales que permitan la mejora de las relaciones entre iguales, así como la adquisición de los contenidos propios de la asignatura. Además, destacan otros estudios que apuestan por la implementación de determinadas estrategias de gamificación, combinándolas con la puesta en práctica de metodologías activas en Educación Superior como pueda ser "Flipped Classroom". 
Tabla 2

Resumen de los artículos incluidos en la revisión sistemática ordenados cronológicamente

\begin{tabular}{|c|c|c|c|c|}
\hline & Estudio & Contexto & $\begin{array}{l}\text { Herramienta de } \\
\text { gamificación }\end{array}$ & Citas \\
\hline 1 & Pettit et al. (2015) & $\begin{array}{l}\text { Escuela de Medicina Os- } \\
\text { teopática de Arizona (Esta- } \\
\text { dos Unidos) }\end{array}$ & $\begin{array}{l}\text { Juego de encuestas: Turn- } \\
\text { ingPoint }\end{array}$ & 19 \\
\hline 2 & Pérez y Rivera (2017) & $\begin{array}{l}\text { Grado en Ciencias de la Ac- } \\
\text { tividad Física y del Deporte. } \\
\text { Universidad de Granada } \\
\text { (España) } \\
\end{array}$ & $\begin{array}{l}\text { Juego de rol: “Juramento } \\
\text { de los elegidos" }\end{array}$ & 1 \\
\hline 3 & $\begin{array}{l}\text { Aynsley, Nathawat y } \\
\text { Crawford (2018) }\end{array}$ & $\begin{array}{l}\text { Estudiantes de Medicina. } \\
\text { Keele University (Reino } \\
\text { Unido) }\end{array}$ & $\begin{array}{l}\text { Juego de roles en equipo: } \\
\text { Braincept }\end{array}$ & 3 \\
\hline 4 & $\begin{array}{l}\text { Głowacki, Kriujova y } \\
\text { Avshenyuk (2018) }\end{array}$ & $\begin{array}{l}\text { Curso de lengua inglesa. } \\
\text { Universidad Técnica Nacio- } \\
\text { nal (Ucrania) }\end{array}$ & $\begin{array}{l}\text { Cuestionario de elección } \\
\text { múltiple: Kahoot! }\end{array}$ & 2 \\
\hline 5 & $\begin{array}{l}\text { Santos, De Souza y De } \\
\text { Sousa (2018) }\end{array}$ & $\begin{array}{l}\text { Grado en Informática, Min- } \\
\text { ería y Agricultura. Instituto } \\
\text { Federal de Piauí (Brasil) }\end{array}$ & $\begin{array}{l}\text { Red social MEIO para la } \\
\text { educación ambiental }\end{array}$ & - \\
\hline 6 & Tan (2018) & $\begin{array}{l}\text { Centro de Comunicación de } \\
\text { Inglés. Universidad Nacio- } \\
\text { nal de Singapur (Malasia) }\end{array}$ & $\begin{array}{l}\text { Juego digital de lectura: } \\
\text { "The Protégé" }\end{array}$ & - \\
\hline 7 & Barreal y Jannes (2019) & $\begin{array}{l}\text { Grado de Estadística, } \\
\text { Matemáticas, Relaciones } \\
\text { Laborales y Turismo. Uni- } \\
\text { versidad de Sevilla (España) }\end{array}$ & $\begin{array}{l}\text { Juego de simulación Bu- } \\
\text { gaMAP: juego de estrate- } \\
\text { gia empresarial aplicado } \\
\text { al mercado de seguros, } \\
\text { desarrollado por la fun- } \\
\text { dación MAPFRE }\end{array}$ & - \\
\hline 8 & Carrión (2019) & $\begin{array}{l}\text { Grado en Educación Pri- } \\
\text { maria (Mención Música). } \\
\text { Universidad Camilo José } \\
\text { Cela (España) } \\
\end{array}$ & $\begin{array}{l}\text { Recurso de juego educa- } \\
\text { tivo: Celebriti Edu } \\
\text { Cuestionario de elección } \\
\text { múltiple: Kahoot! }\end{array}$ & - \\
\hline 9 & Delport (2019) & $\begin{array}{l}\text { Universidad Central de Tec- } \\
\text { nología (Sudáfrica) }\end{array}$ & $\begin{array}{l}\text { Aplicación para la ense- } \\
\text { nanza de la aritmética: } \\
\text { MindTap Math Founda- } \\
\text { tions }\end{array}$ & - \\
\hline 10 & $\begin{array}{l}\text { De los Ríos, Muñoz, } \\
\text { Castro y Arroyo (2019) }\end{array}$ & $\begin{array}{l}\text { Grado en Administración } \\
\text { y Dirección de Empresas. } \\
\text { Universidad Loyola de An- } \\
\text { dalucía (España) }\end{array}$ & $\begin{array}{l}\text { Concurso-repaso: eIn- } \\
\text { struction Flow } \\
\text { Juego de reclutamiento: } \\
\text { L'Oréal Brandstorm } \\
\end{array}$ & - \\
\hline 11 & Felszeghy et al. (2019) & $\begin{array}{l}\text { Grado en Histología Médica } \\
\text { y Dental. Universidad del } \\
\text { Este (Finlandia) }\end{array}$ & $\begin{array}{l}\text { Cuestionario de elección } \\
\text { múltiple: Kahoot! }\end{array}$ & - \\
\hline
\end{tabular}




\begin{tabular}{|c|c|c|c|c|}
\hline & Estudio & Contexto & \begin{tabular}{|l|}
$\begin{array}{l}\text { Herramienta de } \\
\text { gamificación }\end{array}$ \\
\end{tabular} & Citas \\
\hline 12 & $\begin{array}{l}\text { Grivokostopoulou, } \\
\text { Kovas y Perikos (2019) }\end{array}$ & $\begin{array}{l}\text { Grado en Ingeniería Infor- } \\
\text { mática. Universidad de Pa- } \\
\text { tras (Grecia) }\end{array}$ & $\begin{array}{l}\text { Juego de realidad virtual } \\
\text { 3D para enseñar al estu- } \\
\text { diante a actuar de forma } \\
\text { emprendedora y conver- } \\
\text { tirse en un empresario } \\
\text { con éxito. } \\
\end{array}$ & - \\
\hline 13 & $\begin{array}{l}\text { Jong, Chan, Hue y } \\
\text { Tam (2018) }\end{array}$ & $\begin{array}{l}\text { Humanidades y Ciencias } \\
\text { Sociales. Universidad de } \\
\text { Hong-Kong (China) }\end{array}$ & $\begin{array}{l}\text { Aplicación móvil com- } \\
\text { patible con GPS para el } \\
\text { aprendizaje de consultas } \\
\text { sociales en entornos al } \\
\text { aire libre: Gamified Au- } \\
\text { thentic Mobile Enquiry } \\
\text { in Society (GAMES) }\end{array}$ & 3 \\
\hline 14 & $\begin{array}{l}\text { Kapralova, Moskaleva } \\
\text { y Byiyk (2019) }\end{array}$ & \begin{tabular}{|l} 
Grado en Tecnología de la \\
Información. Universidad \\
Politécnica Muadzam Shah \\
Pahang (Malasia) \\
\end{tabular} & $\begin{array}{l}\text { Cuestionarios de elec- } \\
\text { ción múltiple: Kahhot! y } \\
\text { Quizizz }\end{array}$ & - \\
\hline 15 & $\begin{array}{l}\text { López, García y Cer- } \\
\text { vantes (2019) }\end{array}$ & \begin{tabular}{|l|} 
Grado de Ciencias de la Ac- \\
tividad Física de la Universi- \\
dad de Granada (España) \\
\end{tabular} & $\begin{array}{l}\text { Juego de roles: "GamE } \\
\text { oF trhones: la ira de los } \\
\text { dragones" }\end{array}$ & - \\
\hline 16 & Ntokos (2019) & $\begin{array}{l}\text { Grado en Ingeniería Infor- } \\
\text { mática de Southampton } \\
\text { Solent University (Reino } \\
\text { Unido) }\end{array}$ & $\begin{array}{l}\text { Juegos de rol: "Swords } \\
\text { and sorcery" }\end{array}$ & - \\
\hline 17 & $\begin{array}{l}\text { Rahman, Ahmad y } \\
\text { Hashim (2019) }\end{array}$ & $\begin{array}{l}\text { Grado en Tecnología de la } \\
\text { Información de la Universi- } \\
\text { dad Politécnica "Muadzam } \\
\text { Shah Pahang" (Malasia) } \\
\end{array}$ & $\begin{array}{l}\text { Cuestionarios de elec- } \\
\text { ción múltiple: Kahoot! y } \\
\text { Quizizz }\end{array}$ & 3 \\
\hline 18 & $\begin{array}{l}\text { Tóth, Lògó y Lògó } \\
\text { (2019) }\end{array}$ & $\begin{array}{l}\text { Grado en Psicología. Univer- } \\
\text { sidad Tecnológica de Buda- } \\
\text { pest (Hungría) }\end{array}$ & $\begin{array}{l}\text { Cuestionarios de elección } \\
\text { múltiple: Kahoot! }\end{array}$ & 1 \\
\hline 19 & $\begin{array}{l}\text { Zamora, Corrales, Sán- } \\
\text { chez y Espejo (2019) }\end{array}$ & $\begin{array}{l}\text { Grado en Educación Pri- } \\
\text { maria de la Universidad de } \\
\text { Extremadura (España) }\end{array}$ & $\begin{array}{l}\text { Cuestionarios de elección } \\
\text { múltiple: Kahoot, Socra- } \\
\text { tive, Quizziz, etc.; Juegos } \\
\text { de mesa; Une con flechas; } \\
\text { Scaperoom; Café cientí- } \\
\text { fico; Rompecabezas } \\
\end{array}$ & - \\
\hline 20 & $\begin{array}{l}\text { Zvarych, Kalaur, Pry- } \\
\text { machenko, Romash- } \\
\text { chenko y Romanyshy- } \\
\text { na (2019) }\end{array}$ & $\begin{array}{l}\text { Curso de Lengua Extranjera } \\
\text { Profesional para el Grado } \\
\text { en Comercio Internacio- } \\
\text { nal, Derecho, Marketing, } \\
\text { Economía, Gestión y Psi- } \\
\text { cología. Universidad Nacio- } \\
\text { nal de comercio y Economía } \\
\text { de Kiev (Ucrania) }\end{array}$ & $\begin{array}{l}\text { Juego de simulación: } \\
\text { Procedimiento de impor- } \\
\text { tación-exportación de } \\
\text { Café: contenedor LEGO } \\
\text { maersk sealand) }\end{array}$ & 2 \\
\hline
\end{tabular}




\section{Implicaciones de la gamificación para el aprendizaje del estudiante en Educación Superior}

Las investigaciones revisadas plantean la utilización de diferentes herramientas para evaluar el aprendizaje y la adquisición de determinadas competencias en el estudiante, dinamizar los contenidos a desarrollar en el aula, así como favorecer la motivación, el compromiso y la autonomía del estudiante hacia el aprendizaje. Además, en su mayoría utilizan las TIC en el proceso de enseñanza-aprendizaje de los estudiantes en Educación Superior, lo que posibilita la puesta en práctica de videojuegos, aplicaciones informáticas, plataformas webs, etc.

Desde el punto de vista metodológico, las publicaciones revisadas se organizan según dos enfoques diferentes: por un lado, aquellas que ponen en práctica un enfoque de gamificación, el cual permite el manejo de mecánicas de juego en el ámbito educativo y, por otro lado, de Aprendizaje Basado en el Juego, lo que posibilita la utilización de juegos o videojuegos con fines didácticos en Educación Superior. Mayoritariamente, se trata de estudios de carácter cuantitativo (basados en la utilización de encuestas) que incluyen una muestra reducida, al estar desarrolladas en el contexto de una determinada asignatura.

En cuanto a las implicaciones de la gamificación para el aprendizaje del estudiante, éstas se han categorizado según su naturaleza atendiendo a la dimensión cognitiva, procedimental y actitudinal. En el plano cognitivo, cabe destacar cómo la práctica de experiencias de aprendizaje gamificado permite una mejora del rendimiento académico en el estudiante, ayudándolo a maximizar el aprendizaje. A su vez, posibilita una alta transferencia de lo aprendido para su futura práctica profesional, lo que implica la mejora en la capacitación del estudiante, así como la adquisición de determinadas habilidades. Dicha práctica también favorece la construcción colectiva del conocimiento, una mayor confianza en su manejo, así como una mejor asimilación, comprensión y retención del conocimiento. Incluso, cabe destacar una mejora de la satisfacción del estudiante hacia el conocimiento adquirido, al haberse planteado a partir de un proceso basado en la diversión e interacción entre iguales.

En cuanto a la dimensión procedimental, las investigaciones revisadas plantean cómo la práctica de experiencias de aprendizaje gamificado implica un mayor nivel de logro hacia el aprendizaje. De este modo, se refuerza la utilidad del juego para futuras experiencias profesionales en el estudiante, posibilitando el desarrollo de competencias y habilidades demandadas por las organizaciones profesionales. A su vez, se favorece el desarrollo de procesos de aprendizaje activo grupal y de evaluación compartida. También posibilita el refuerzo de contenidos y el desarrollo de habilidades competitivas entre el alumnado, facilitando el análisis de situaciones y la toma de decisiones.

Finalmente, y para la dimensión actitudinal, las investigaciones revisadas ponen de manifiesto cómo el desarrollo de este tipo de práctica docente innovadora aumenta la motivación, autoeficacia, el compromiso y el interés del estudiante hacia la asignatura. Además, favorece la creación de un buen clima de aula, basada en la participación del estudiante hacia el desarrollo de un proceso de aprendizaje divertido, atractivo y colaborativo. También se produce una mejora en el comportamiento del estudiante y el desarrollo de determinadas habilidades interpersonales. 


\begin{tabular}{|c|c|c|c|c|c|c|c|}
\hline & | & 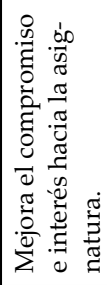 & 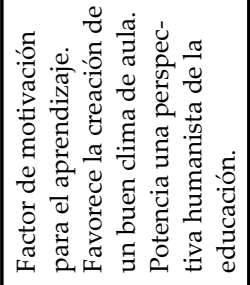 & 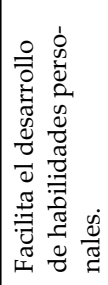 & 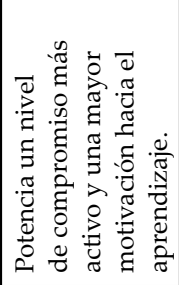 & 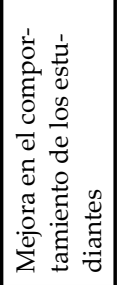 & 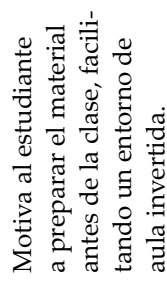 \\
\hline & 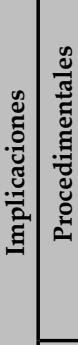 & & 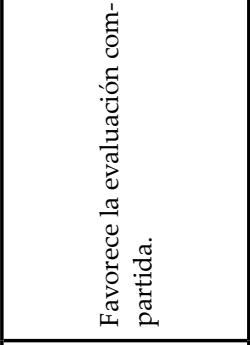 & & 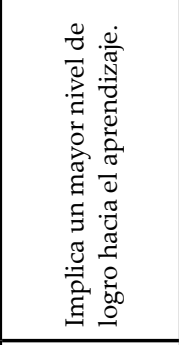 & & \\
\hline \multicolumn{8}{|c|}{ 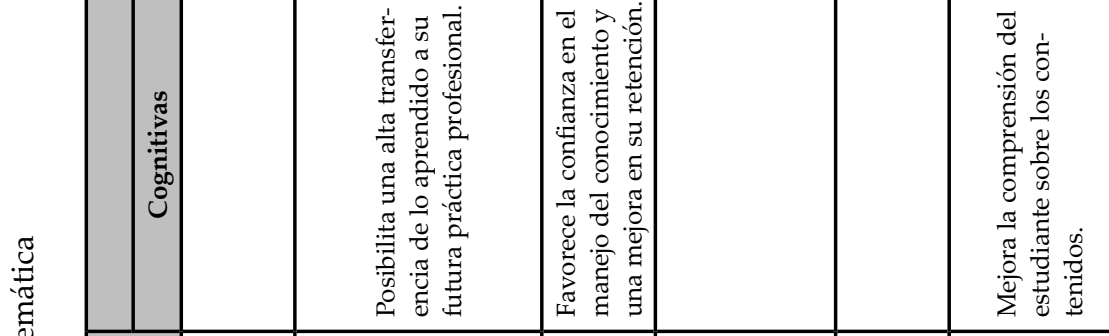 } \\
\hline$\stackrel{n}{\infty}$ & $\stackrel{*}{*}$ & 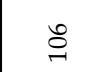 & g & $\stackrel{\llcorner}{\underset{\sim}{\sim}}$ & F & $\underset{\sim}{\infty}$ & ส \\
\hline 离 & & 忘 & 完 & 恴 & 忘 & 忘 & 怠 \\
\hline 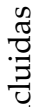 & 苞 & $\sum_{0}$ & 品 & $\overrightarrow{00}$ & $\sum_{0}$ & 品 & 品 \\
\hline 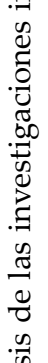 & 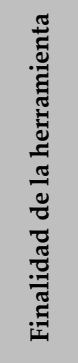 & 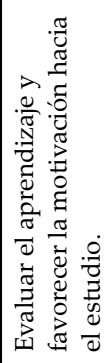 & 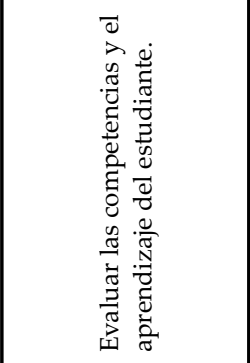 & 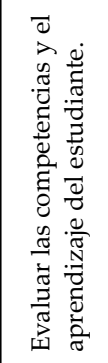 & 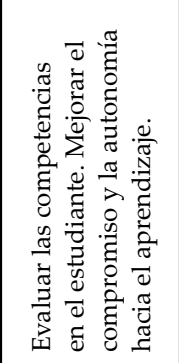 & 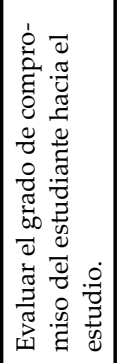 & 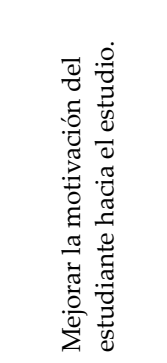 \\
\hline is & & 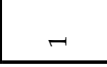 & $N$ & 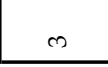 & + & 10 & 0 \\
\hline
\end{tabular}




\begin{tabular}{|c|c|c|c|c|c|c|c|}
\hline 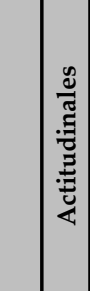 & 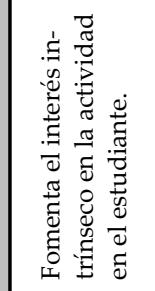 & 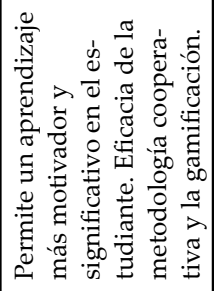 & 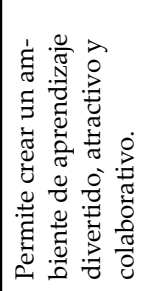 & 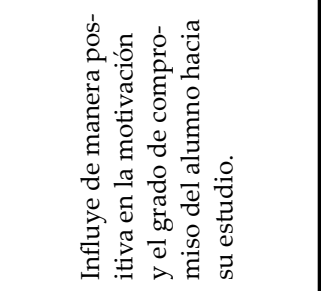 & 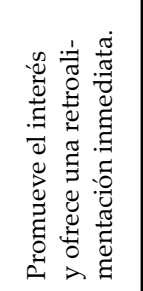 & 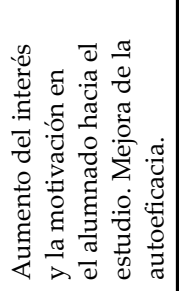 & 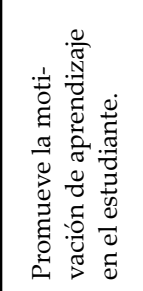 \\
\hline 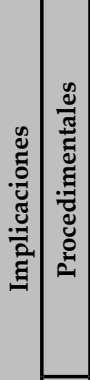 & 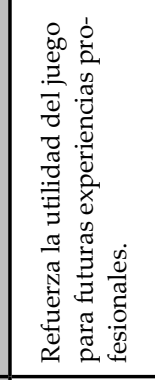 & & 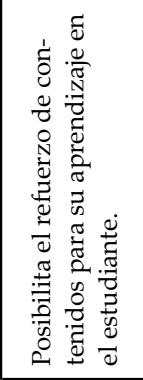 & 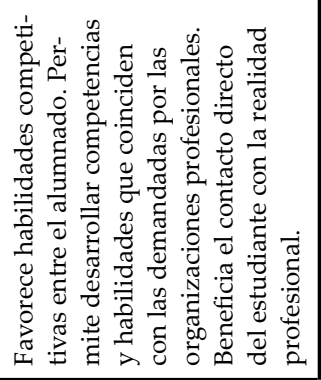 & 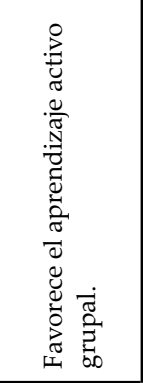 & 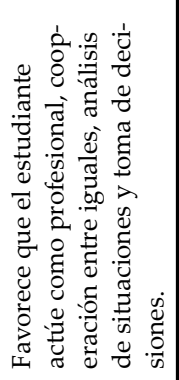 & \\
\hline 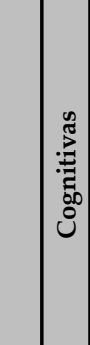 & 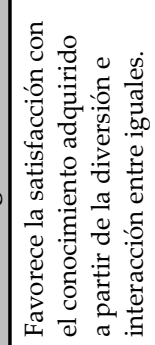 & 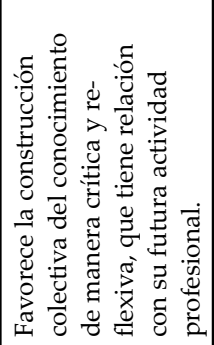 & 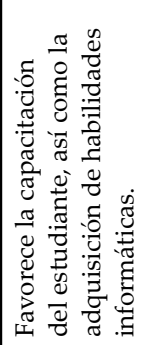 & 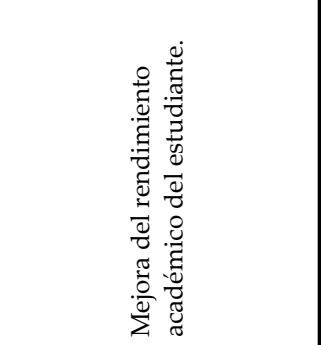 & 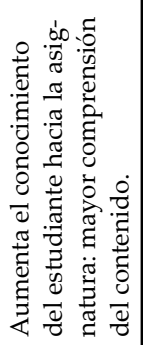 & 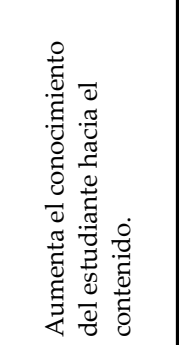 & 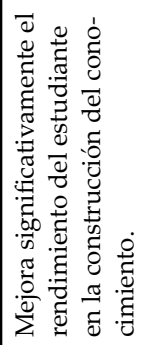 \\
\hline$\stackrel{*}{*}$ & $\underset{\exists}{\mathcal{I}}$ & $\stackrel{\text { L }}{\circ}$ & $\underset{\exists}{\stackrel{8}{\sigma}}$ & $\stackrel{\mathscr{L}}{\mathfrak{I}}$ & $\stackrel{\mathscr{N}}{\sim}$ & $\infty$ & ถิ \\
\hline 莡 & 莣穵 & 究忩 & 忘 & 空 & 究 & 空 & 空 \\
\hline * & 䓽 & $\overrightarrow{0}$ & 品 & जै & 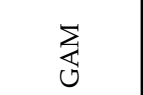 & 品 & 苍 \\
\hline 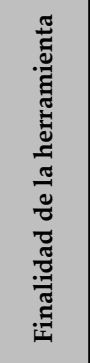 & 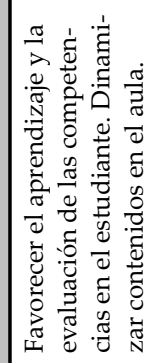 & 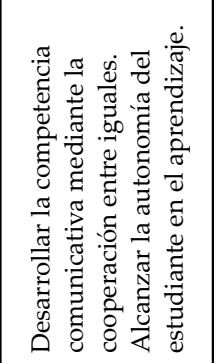 & 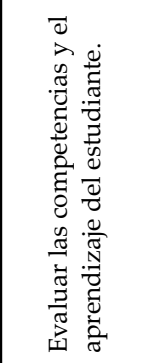 & 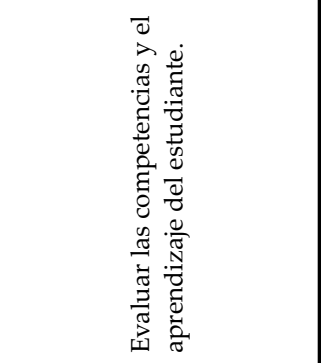 & 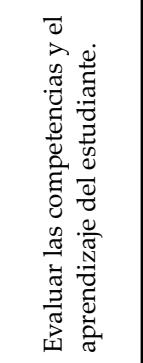 & 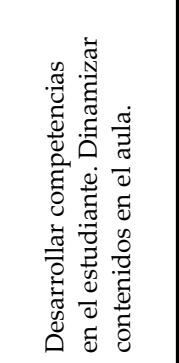 & 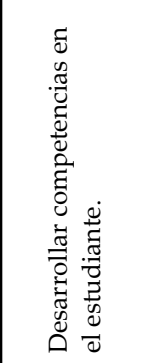 \\
\hline & $\Lambda$ & $\infty$ & $a$ & 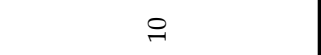 & $F$ & $\approx$ & $\stackrel{m}{\sim}$ \\
\hline
\end{tabular}




\begin{tabular}{|c|c|c|c|c|c|c|c|}
\hline 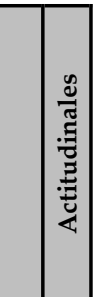 & 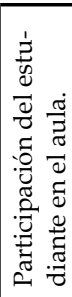 & 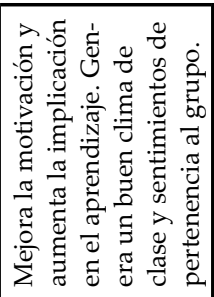 & 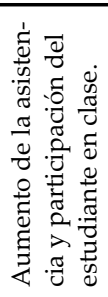 & 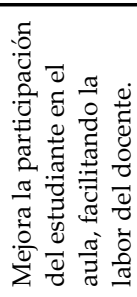 & 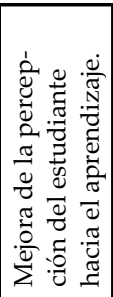 & 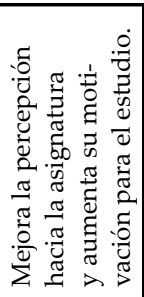 & 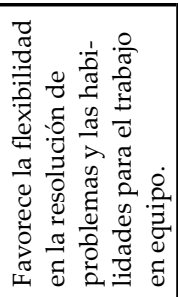 \\
\hline 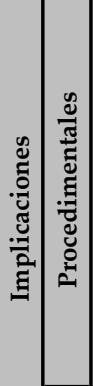 & & & & & & 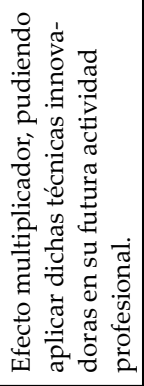 & \\
\hline 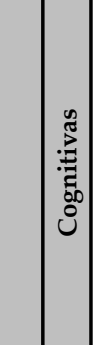 & 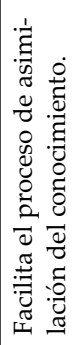 & & 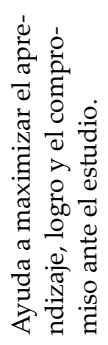 & & 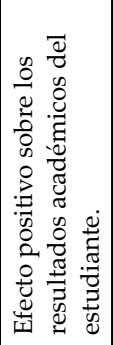 & & 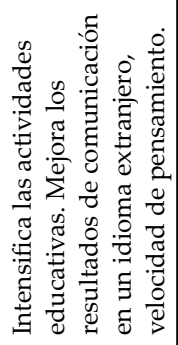 \\
\hline$\stackrel{*}{*}$ & in & in & ले & in & ڤ્సે & $\stackrel{\infty}{\sim}$ & டீ \\
\hline$\stackrel{\stackrel{*}{*}}{\stackrel{*}{*}}$ & 峞 & 忘 & 峞 & 忘 & 忘 & 空空 & 岕 \\
\hline 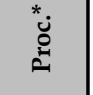 & 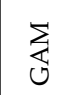 & $\overrightarrow{0}$ & $\dot{0}$ & 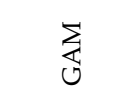 & 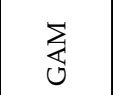 & $\sum_{0}$ & 苍 \\
\hline 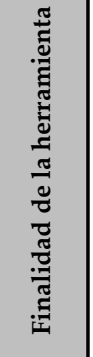 & 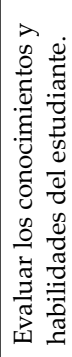 & 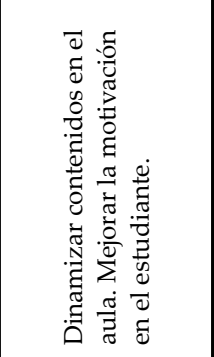 & 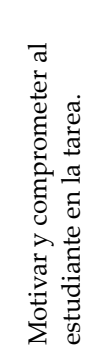 & 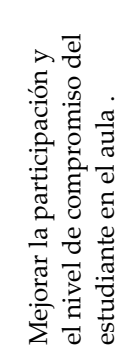 & 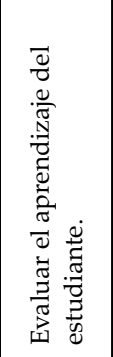 & 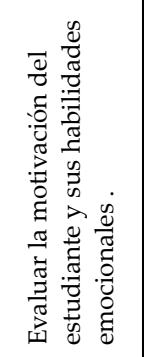 & 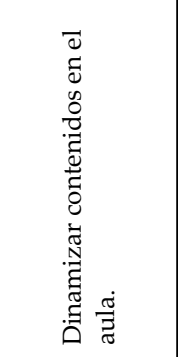 \\
\hline & $\exists$ & $\stackrel{12}{\sim}$ & $\stackrel{?}{2}$ & 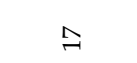 & $\stackrel{\infty}{=}$ & 2 & ন \\
\hline
\end{tabular}




\section{Discusión y conclusiones}

Este trabajo se centra en una estrategia metodológica que favorece el desarrollo de metodologías activas en el proceso didáctico, permitiendo aunar dos conceptos diferentes: juego y aprendizaje. Resulta de interés conocer el significado que adquiere la gamificación para el estudiante universitario, pues el profesorado debe lograr su compromiso con dicho proceso (Abdul \& Felicia, 2015; Huang et al., 2019).

Con la intención de responder al objetivo 1, este trabajo demuestra el interés creciente entre la comunidad científica por plantear investigaciones y trabajos científicos sobre gamificación en Educación Superior. En su mayoría, dichas propuestas plantean experiencias de innovación educativa vinculadas con titulaciones adscritas a las áreas de conocimiento de Ciencias Sociales y Jurídicas, Ingeniería y Arquitectura y Ciencias de la Salud, siendo la titulación de Ingeniería Informática la que mayor frecuencia de uso registra.

En cuanto a la relación entre el concepto de gamificación y el área de Ingeniería, cabe destacar la proliferación de distintas herramientas de gamificación mediadas por el uso de las TIC, lo que justifica la elevada implicación del profesorado adscrito a esta área de conocimiento hacia el uso de este tipo de recursos didácticos en el aula universitaria. De igual modo, y en el ámbito educativo, se ha utilizado tradicionalmente determinados elementos del juego para facilitar el proceso de aprendizaje al estudiante en todas las etapas educativas. Finalmente, la vinculación de la gamificación con el área de Ciencias Sociales y Jurídicas puede justificarse dado que el concepto de gamificación surge en el sector de las empresas y el marketing, habiéndose aplicado a otros contextos con mucha rapidez.

Desde la Educación Superior, se debe aprovechar el interés social por la utilización de este tipo de herramientas, implementándolas en el proceso didáctico. En este sentido, Aldemir et al. (2018) demuestran la necesidad del profesorado de diseñar el juego de manera adecuada, estableciéndose como criterio que justifica el alto grado de aceptación del estudiante hacia estas experiencias. Así pues, lo relevante no es el juego en sí mismo, sino la metodología utilizada para el uso del juego (Rodríguez-Hoyos \& Joao, 2013) a partir de dinámicas centradas en el diseño de retos, recompensas y logros y el desarrollo de incentivos intrínsecos en el estudiante (Kokkalia et al., 2017). Ello requiere del esfuerzo del profesorado, pues las experiencias de gamificación propuestas al estudiante deben estar planificadas previamente y ligadas a objetivos didácticos concretos, debiendo plantearse para un determinado contenido y según las características del grupo de estudiantes al que se dirige.

Atendiendo al objetivo 2, las investigaciones revisadas demuestran la puesta en práctica de diferentes experiencias de innovación docente en Educación Superior. En ellas, se utiliza el juego como elemento transversal para la evaluación del aprendizaje y la adquisición de determinadas competencias en el estudiante, la dinamización de los contenidos a desarrollar en el aula y un aumento de la motivación y el interés del estudiante. Sin duda, se trata de herramientas pertinentes para facilitar el desarrollo de contenidos y mejorar el interés y la participación del estudiante ante el proceso de enseñanza-aprendizaje (Corchuelo, 2018; Villalustre \& Del Moral, 2015). 
Para conseguir tales objetivos, las investigaciones revisadas utilizan dos enfoques diferentes: por un lado, las estrategias de gamificación que permiten la puesta en práctica de dinámicas y mecánicas de juego en un entorno que no es lúdico, como es el ámbito educativo. Por otro lado, el Aprendizaje Basado en el Juego (GBL) que utiliza determinados juegos para facilitar la adquisición de ciertos conocimientos y habilidades en el estudiante. Sin embargo, y a pesar de utilizar dos enfoques diferentes, todas las publicaciones revisadas sitúan al juego como elemento transversal del proceso didáctico con la intención de mejorar la práctica docente y modificar las actitudes y aptitudes dentro de los individuos involucrados en el juego (Hernández, Monroy \& Jiménez, 2018).

En cuanto a las implicaciones de este tipo de prácticas innovadoras en la Educación Superior, cabe establecer una triple clasificación según su naturaleza: ámbito cognitivo (Aynsley et al., 2018; Barreal \& Jannes, 2019; De los Ríos et al., 2019; Felszeghy et al. 2019; Jong et al., 2018; Tan, 2018; Tóth et al. 2019; Zvarych et al., 2019), dimensión procedimental (Barrel \& Jannes, 2019; De los Ríos et al., 2019; Delport, 2019; Felszeghy et al., 2019: Glowacki et al., 2018; Grivokostopoulou et al., 2019: Pérez \& Rivera, 2017) y actitudinal (Głowacki et al., 2018; Grivokostopoulou et al., 2019; Kapralova et al., 2019; López et al., 2019; Pettit et al., 2015; Santos et al., 2018).

Estos resultados guardan relación con los aportados en otras investigaciones que revelan cómo los estudiantes involucrados en entornos de aprendizaje gamificados mejoran su aprendizaje y rendimiento académico (Marín, Frez, Cruz \& Genero, 2019) a la vez que favorece su predisposición para adquirir determinadas competencias (Kapp, 2012; Zichermann \& Cunningham, 2011). Otros estudios, además, informan de las implicaciones de la gamificación hacia un aumento del nivel de motivación y compromiso del estudiante (Chu \& Hung, 2015; García, Carrascal \& Renovell, 2016), así como su participación en el proceso de aprendizaje (Alhammad \& Moreno, 2018; Çakıroğlu et al., 2017).

En síntesis, esta revisión bibliográfica demuestra la percepción favorable del estudiante universitario hacia el desarrollo de experiencias didácticas innovadoras basadas en la gamificación. Ello refuerza la necesidad de incrementar la presencia de estas prácticas docentes innovadoras en Educación Superior, dejando atrás experiencias aisladas para asignaturas e incluso, contenidos concretos. El desarrollo de estrategias didácticas de gamificación debe ser trasversal al proceso didáctico planteado por el profesorado, lo que requiere de un análisis inicial que examine el punto de partida de los agentes educativos implicados.

Sin duda, se trata de un trabajo de interés para el profesorado universitario que pretenda iniciarse en la práctica de esta metodología innovadora en el aula, Sin embargo, entre las limitaciones de este trabajo, se encuentra el escaso número de investigaciones que plantean experiencias de gamificación a partir de recursos sin soporte digital, lo que invita a cuestionar la viabilidad de estos hallazgos para este tipo de herramientas. Como futura línea de investigación, se plantea indagar las percepciones del profesorado hacia la práctica de estrategias de gamificación en el aula universitaria, pues junto al estudiante, son los principales agentes implicados en el proceso didáctico. 


\section{Referencias}

Abdul, A.I., \& Felicia, P. (2015). Gameplay engagement and learning in game-based learning: A systematic review. Review of educational research, 85(4), 740-779. doi: 10.3102/0034654315577210

Aldemir, T., Celik, B., \& Kaplan, G. (2018). A qualitative investigation of student perceptions of game elements in a gamified course. Computers in Human Behavior, 78, 235-254. doi: 10.1016/j.chb.2017.10.001

Alexander, P.A. (2020). Methodological guidance paper: The art and science of quality systematic reviews. Review of Educational Research, 90(1), 6-23. doi: 10.3102/0034654319854352

Alhammad, M.M. \& Moreno, A.M. (2018). Gamification in software engineering education: A systematic mapping. Journal of Systems and Software, 141, 131-150. doi: 10.1016/J.JSS.2018.03.065

Alsawaier, R.S. (2018). The effect of gamification on motivation and engagement. The International Journal of Information and Learning Technology, 35(1), 56-79. doi: 10.1108/ IJILT-02-2017-0009

Ardila, J. (2019). Supuestos teóricos para la gamificación de la educación superior. Magis, Revista Internacional de Investigación en Educación, 12(24), 71-84. doi: 10.11144/ Javeriana.m12-24.stge

Aynsley, S.A., Nathawat, K., \& Crawford, R.M. (2018). Evaluating student perceptions of using a game-based approach to aid learning: Braincept. Higher Education Pedagogies, 3(1), 478-489. doi: 10.1080/23752696.2018.1435296

Barreal, J., \& Jannes, G. (2019). Narrative as a teaching tool in the gamification of statistics in the Degree of Tourism. Digital Education Review, 36, 152-170.

Bodnar, C.A., Anastasio, D., Enszer, J.A., \& Burkey, D.D. (2016). Engineers at play: Games as teaching tools for undergraduate engineering students. Journal of Engineering Education, 105(1), 147-200. doi: 10.1002/jee.20106

Borrego, C., Fernández, C., Blanes, I., \& Robles, S. (2017). Room escape at class: Escape games activities to facilitate the motivation and learning in computer science. JOTSE: Journal of Technology and Science Education, 7(2), 162-171. doi: 10.3926/jotse.247

Brull, S., \& Finlayson, S. (2016). Importance of gamification in increasing learning. The Journal of Continuing Education in Nursing, 47(8), 372-375. doi: 10.3928/0022012420160715-09

Çakıroğlu, Ü, Başıbüyük, B., Güler, M., Atabay, M., \& Yilmaz, B. (2017). Gamifying an ICT course: Influences on engagement and academic performance. Computers in Human Behavior, 69, 98-107. doi: 10.1016/J.CHB.2016.12.018

Carrión, E. (2019). The use of the game and the cooperative Methodology in Higher Education: An alternative for creative teaching. Artseduca, 23, 71-97. doi: 10.6035/ Artseduca.2019.23.4

Chaiyo, Y., \& Nokham, R. (2017). The effect of Kahoot, Quizizz and Google Forms on the student's perception in the classrooms response system. Proceedings of the International Conference on Digital Arts, Media and Technology (ICDAMT). Chiang Mai (Thailand). doi: 10.1109/ICDAMT.2017.7904957 
Chu, C. \& Hung, H. (2015). Effects of the Digital Game-Development Approach on Elementary School Students` Learning Motivation, Problem Solving, and Learning Achievement. International Journal of Distance Education Technologies, 13(1), 87-102. doi: 10.4018/ijdet.2015010105

Contreras, R., \& Eguia, J.L. (2016). Gamificación en aulas universitarias. Bellaterra: Institut de la Comunicació.

Corchuelo, C.A. (2018). Gamificación en Educación Superior: experiencia innovadora para motivar estudiantes y dinamizar contenidos en el aula. EDUTEC, 63, 29-41. doi: 10.21556/edutec.2018.63.927

Dascalu, M.I., Tesila, B., \& Nedelcu, R.A. (2016). Enhancing employability through e-learning communities: From myth to reality. In Y. Li, M. Kravcik, E. Pospecu, R. Huang, N. Kinshuk, \& N.S. Chen (Coords.). State-of-the-art and future directions of smart learning (pp. 309-313). Singapore: Springer. doi: 10.1007/978-981-287-868-7_38

De los Ríos, A., Munoz, Y., Castro, P., \& Arroyo, J.L. (2019). Gamification, strategy shared between university, Company and millennials. Revista de Docencia Universitaria, 17(2), 73-88. doi: 10.4995/redu.2019

Delport, D.H. (2019). Numeracy students ' perspectives on a new digital learning tool at a South African University. South African Journal of Higher Education, 33(5), 25-41. doi: $10.20853 / 33-5-3588$

Dicheva, D., Dichev, C., Agre, G., \& Angelova, G. (2015). Gamification in education: A systematic mapping study. Journal of Educational Technology E Society, 18(3), 75-88. Recuperado de: https://cutt.ly/jyFsnhS

Felszeghy, S., Pasonen-Seppänen, S., Koskela, A., Nieminen, P., Härkönen, K., Paldanius, K.M., ... \& Haapaniemi, T. (2019). Using online game-based platforms to improve student performance and engagement in histology teaching. BMC medical education, 19(1), 273-284. doi: 10.1186/s12909-019-1701-0

García, F., Carrascal, S. \& Ronobell, V. (2016). The drawing of the human figure "Avatar" as an element for the development of creativity and learning through gamification techniques in Primary Education. ArDIn. Arte, Diseño e ingeniería, 5, 47.57. Recuperado de: https://cutt.ly/QyLL8YP

Gay, A.S., \& Burbridge, L. (2016). "Bring your own device” for formative assessment. The Mathematics Teacher, 110(4), 310-313. doi: 10.5951/mathteacher.110.4.0310

Głowacki, J., Kriukova, Y., \& Avshenyuk, N. (2018). Gamification in higher education: experience of Poland and Ukraine. Advanced Education, 5(10), 105-110. doi: $10.20535 / 2410-8286.151143$

Grivokostopoulou, F., Kovas, K., \& Perikos, I. (2019). Examing the impact of a gamified entrepreneurship education framework in higher education. Sustainability, 11, 56235640. doi: 10.3390/su11205623

Hanus, M., \& Fox, J. (2015). Assessing the effects of gamification in the classroom: A longitudinal study on intrinsic motivation, social comparison, satisfaction, effort and academic performance. Computers E Education, 80, 152-161. doi: 10.1016/j. compedu.2014.08.019

Herrera, L., Souza, M.R., \& Soares, J.F. (2018). Evaluación de la calidad de la educación superior: una revisión de la literatura a partir de la satisfacción del alumnado. Cadernos Pesquisa, 25(2), 71-90. Recuperado de: https://cutt.ly/lyFsYC8 
Howel, D.D., Tseng, D., \& Colorado, J.T. (2017). Fast assessments with digital tools using multiple-choice questions. College Teaching, 1-3, 145-147. doi: 10.1080/87567555.2017.1291489.

Huang, B., Hew, K.F., \& Lo, C.K. (2019). Investigating the effects of gamification-enhanced flipped learning on undergraduate students' behavioral and cognitive engagement. Interactive Learning Environments, 27(8), 1106-1126. doi: 10.1080/10494820.2018.1495653

Jong, M., Chan, T., Hue, M.T., \& Tam, V. (2018). Gamifying and Mobilising Social Enquiry-based Learning in authentic outdoor environments. Educational Technology $\mathcal{E}$ Society, 21(4), 277-292. Recuperado de: https://cutt.ly/ayFsUNn

Kapp, K.M. (2012). The Gamification of Learning and Instruction: Game-Based Methods and Strategies for Training and Education. San Francisco, CA: John Wiley

Kapralova, J.V., Moskaleva, L.A., \& Byiyk, I.A. (2019). Learning the Russian language in the game: Traditional and new approaches. International Journal of Higher Education, 8(7), 50-55. doi: 10.5430/ijhe.v8n7p50

Ke, F. (2014). An implementation of design-based learning through creating educational computer games: A case study on mathematics learning during design and computing. Computers \& Education, 73, 26-39. doi: 10.1016/j.compedu.2013.12.010

Kokkalia, G., Drigas, A., Roussos, P., \& Economou, A. (2017). Gamifying education: what is known, what is believed and what remains uncertain: a critical review. International Journal of Educational Technology in Higher Education, 14(1), 1-36. doi: 10.1186/s41239-017-0042-5.

López, I.P., García, E.R., \& Cervantes, C.T. (2019). 12+1. Feelings of physical education college students towards a gamification proposal: "game of thrones: the anger of the dragons". Movimiento, 25(1), 1-15. doi: 10.22456/1982-8918.88031

Lozada, C., \& Betancur, S. (2017). La gamificación en la educación superior: una revisión sistemática. Revista Ingenierías Universidad de Medellín, 16(31), 97-124. doi: 10.22395/ rium.v16n31a5

Marín, B., Frez, J., Cruz, J., \& Genero, M. (2019). An Empirical Investigaion on the Benefits of Gamification in Programming Courses. ACM Transactions on Computing Education, 19(1), 1-22. doi: 10.1145/3231709

Medvedovska, D., Skarlupina, Y., \& Turchyna, T. (2016). Integrating online educational applications in the classroom. European Humanities Studies: State and Society, 4, 145156. Recuperado de: https://cutt.ly/3yFsOdu

Moffat, D., Farrell, D., Gardiner, B., McCulloch, A., \& Fairlie, F. (2015). A serious game to give students careers advice, awareness and action. In ECEL2015-14th European Conference on e-Learning: ECE12015 (p. 396). Hatfield, UK: Academic Conferences and Publishing Limited.

Ntokos, K. (2019). Swords and sorcery: a structural gamification framework for higher education using role-playing game elements. Research in Learning Technology, 27, 2272. doi: 10.25304/rlt.v27.2272

Oliva, H. (2017). La gamificación como estrategia metodológica en el contexto educativo universitario. Realidad y Reflexión, 44, 29-47. doi: 10.5377/ryr.v44i0.3563

Pascuas, Y.S., Vargas, E.O., \& Muñoz, J.I. (2017). Experiencias motivacionales gamificadas: una revisión sistemática de literatura. Innovación educativa, 17(75), 63-80. Recuperado de: https://cutt.ly/8yFsPsU 
Pérez, A., \& Almela, J. (2018). Gamification and Transmedia for Scientific Promotion and for Encouraging Scientific Careers in Adolescents. Comunicar: Media Education Research Journal, 26(55), 93-103. doi: 10.3916/C55-2018-09

Pérez, I., \& Rivera, E. (2017). Formar docentes, formar personas: análisis de los aprendizajes logrados por estudiantes universitarios desde una experiencia de gamificación. Signo y Pensamiento, 36(70), 112-129. doi: 10.11144/Javeriana.syp36-70.fdfp.

Pettit, R. K., McCoy, L., Kinney, M., \& Schwartz, F.N. (2015). Student perceptions of gamified audience response system interactions in large group lectures and via lecture capture technology. BMC medical education, 15(1), 92. doi: 10.1186/s12909-015-0373-7

Pivec, M. \& Dziabenko, O. (2004). Gamed-Based Learning in Universities and Lifelong Learning: "Unigame: Social Skills and Knowledge Training" Game Concept. Journal of Universal Computer Science, 10(1), 14-26.

Prieto, J.M. (2018). Gamificación del aprendizaje y motivación en universitarios. Elaboración de una historia interactiva: MOTORIA-X. Edutec. Revista Electrónica De Tecnología Educativa, (66), 77-92. doi: 10.21556/edutec.2018.66.1085

Rahman, R.A., Ahmad, S., \& Hashim, U.R. (2018). The effectiveness of gamification technique for higher education student's engagement in polytechnic Muadzam Shah Pahang, Malaysia. International Journal of Educational Technology in Higher Education, 15(1), 41. doi: 10.1186/s41239-018-0123-0

Rodríguez-Espinar, S. (2018). La Universidad: una visión desde "fuera" orientada al futuro. Revista de Investigación Educativa, 36(1), 15-38. doi: 10.6018/rie.36.1.309041

Rodríguez-Hoyos, C., \& Joao, M. (2013). Videojuegos y educación: una visión panorámica de las investigaciones desarrolladas a nivel internacional. Revista de Currículum y Formación del Profesorado, 17(2), 479-494. Recuperado de: https://cutt.ly/3yFsAOO

Sánchez, J., Cañada, F., \& Dávila, M.A. (2017). Just a game? Gamifying a general science class at university: Collaborative and competitive work implications. Thinking skills and creativity. Elsevier Science Direct, 26, 51-59. Recuperado de: https://cutt.ly/WyFsSTk

Santos, M.L., De Souza, R.N., \& De Sousa, M.C. (2018). A gamificação como estratégia de engajamento para a prática da educação ambiental. REMEA-Revista Eletrônica do Mestrado em Educação Ambiental, 35(1), 279-295. doi: 10.14295/remea.v35i1.7519

Tan, Y.L. (2018). Meaningful gamification and students' motivation: A strategy for scaffolding reading material. Online Learning Journal, 22(2), 141-156. doi: 10.24059/olj.v22i2.1167

Torres, A., Ramírez, M.S. \& Romero, L.M. (2018). Valoración y evaluación de los Aprendizajes Basados en Juegos (GBL) en contextos e-learning. Education in the Knowledge Society, 19(4), 109-128. doi: 10.14201/eks2018194109128

Tóth, A., Lógó, P., \& Lógó, E. (2019). The Effect of the Kahoot Quiz on the Student's Results in the Exam. Periodica Polytechnica Social and Management Sciences, 27(2), 173179. doi: 10.3311/PPso.12464

Urrútia, G., \& Bonfill, X. (2010). Declaración PRISMA: una propuesta para mejorar la publicación de revisiones sistemáticas y metaanálisis. Medicina clínica, 135(11), 507511. Recuperado de: https://cutt.ly/qyFsDKJ

Villalustre, L. \& Del Moral, M. (2015). Gamificación: Estrategia para optimizar el proceso de aprendizaje y la adquisición de competencias en contextos universitarios. Digital Education Review, 27, 13-31. doi: 10.1344/der.2015.27.13-31 
Zamora, F., Corrales, M., Sánchez, J., \& Espejo, L. (2019). Nonscientific University Students Training in General Science Using an Active-Learning Merged Pedagogy: Gamification in a Flipped Classroom. Education Sciences, 9, 297-315. doi: 10.3390/educsci9040297

Zichermann, G. \& Cunningham, C. (2011). Gamification by Design: Implementing Game Mechanics in Web and Mobile Apps. Cambridge, MA: O'Reilly Media.

Zvarych, I., Kalaur, S.M., Prymachenko, N.M., Romashchenko, I.V., \& Romanyshyna, O.I. (2019). Gamification as a Tool for Stimulating the Educational Activity of Students of Higher Educational Institutions of Ukraine and the United States. European Journal of Educational Research, 8(3), 875-891. doi: 10.12973/eu-jer.8.3.875

Fecha de recepción: 22 de marzo de 2020.

Fecha de revisión: 8 de abril de 2020.

Fecha de aceptación: 26 de junio de 2020. 